\title{
THE VALUE OF MANIPULATION IN THE TREATMENT OF RHEUMATIC DISEASES.
}

\author{
By JAMES MENNELL, M.D. (Cantab.) \\ (Consulting Physico-therapist, St. Thomas's Hospital; Vice-president and late Chairman of the \\ Council of the Chartered Society of Massage and Medical Gymnastics.)
}

When considering the value of manipulation in the treatment of rheumatic diseases, it is well-nigh essential to divide the problem into two separate sections. First, there are those cases in which active inflammation has involved the joints and in which trauma is playing a subsidiary part. The second group of cases consist of those in which the diagnosis of "rheumatism" is often made, but in which this diagnosis is entirely erroneous. For the purpose of this paper the word " manipulation" will be considered as being applied to manipulation of joints, and little or nothing will be said of the no less important matter, the manipulation of the soft parts. In practice the two are inseparable, but the problem before us now is to estimate the value of administrating forced movement to a joint.

\section{When to mobilise.}

The late Mr. Trethowen always used to say: "When in doubt, move," but before we accept this principle, it is essential to know how we are to decide whether we should be in doubt or not. In other words what we really want to know in the first instance is the answer to the question:-Are there any positive indications that manipulation should not be performed? and fortunately there are several.

The first and foremost is the problem as to whether the condition of the joint has been caused by, or aggravated by, a known or suspected focus of infection. In other words, before considering whether to manipulate the joint or not, the first thing to decide is whether the joint condition of the patient justifies it. One example might serve by way of illustration. Some years ago a patient was complaining of severe backache which, as a result of examination, proved to be due to pain on movement of the joints of the lower lumbar spine. I suspected a focus of infection, examined the mouth and felt very suspicious about the condition of his teeth. I told him I would not dream of manipulating until I had assurance from his dentist that there was no focus of infection at the roots of his teeth. He then stated that he had received this assurance only a few days before: I therefore manipulated and the condition flared up very materially. I told him that there must be a focus of infection somewhere, and he then informed me that he thought perhaps he had better take my advice and see his dentist, as he had deliberately deceived me when he said that he had already done so. The dental extraction led to complete recovery within a few months.

It is plain from this story that sometimes we should be justified, when in doubt, in using manipulation of a joint as a means of forming a diagnosis. Recently a young naval officer complained of a stiff neck with rheumatic pain radiating down both arms. He had just had some mild infective process in the form of " influenza." The joints of the cervical spine were stiff and the condition found would indicate the possibility at least that the pain radiating down the arms (this was diagnosed as brachial neuritis) was really pain referred from the joints. From the history it was fairly obvious that the joints had suffered from the general infection and the problem was whether the infection was quiescent or still active; it seemed impossible to judge from the history, from the description 
of symptoms or from examination. There was only one way to find out and that was to see what the result of manipulation would be. I therefore put the stiffened joints through about a third of their normal range of movement and stopped. Next day pain was very severe, and three or four days had to pass before the effect of the manipulation wore off. This was enough to prove that active inflammation was still present, and a search was made for a focus which might have been stirred up by the influenza and which had not become quiescent. This case was a particularly troublesome one as the discovery of one focus led to the discovery of another, and this again to a third. He went through a very rough time until the active infections had been adequately treated; then recovery began to take place, but the stiffness remained and the pain down the arms as a result of movement also remained to a very crippling extent. Two months after the removal of the third focus of infection I did exactly the same manipulation as I had done on the former occasion. Not only was there no reaction, but the feeling of relief was instantaneous. There was no reaction (in spite of the fact that the patient in his own mind was convinced that there would be repetition of his former experiences), and after two further and considerably more vigorous manipulations, all symptoms entirely disappeared. Here was a case then in which joint manipulation was used in the first case to establish diagnosis, and second, to remedy the pathological condition. Fortunately however there are as a rule certain signs which should serve as a definite guide that manipulation is injudicious.

In cases where acute symptoms have arisen, either without any history of trauma or with a history in which the trauma mentioned is obviously totally inadequate, suspicion is at once aroused. It is true that difficulties may often arise. I well remember a boy who was sent to see me suffering from a sudden and acute inflammatory condition of the shoulder which came on during the night. The diagnosis of acute fibrositis had been made, but the fact that the shoulder was dislocated had been overlooked. The explanation became obvious in a few days when the patient had his second epileptic fit. The first one occurred at night during sleep and he had dislocated his shoulder during it.

\section{Contra-Indications to mobilisation.}

Active inflammation. There are usually definite danger signals which should always be regarded. Redness, heat, and tenderness over the region of the joint capsule should invariably be regarded as danger signals, but after all comparatively few of our joints are sufficiently near enough to the surface for redness to be visible. Many also are too deep for us to be perfectly certain that it is the transmission of pressure direct to the capsule that causes pain; while there are countless other joints where anything resembling a direct approach is well-nigh impossible. Diagnosis must depend on inference drawn from history and exarnination of the movements of the joint.

Then in examining the movement of the joints in the lower part of the back, one patient may be found to stoop forward to a certain extent with perfect freedom and then the trunk may be noticed to lean over towards the left and the further the patient tries to stoop the further does the deviation extend. The next patient may exhibit exactly. the same signs throughout the early part of the movement, but then, instead of the deviation to the left increasing with the increased range of movement, it will be noticed that the deviation will be reduced again after a certain stage of movement has been passed. On the return journey the same deviation in one position is noticed. As a general rule this may be taken to 
indicate the presence of active inflammation and the patient moves in this manner in order to avoid laying strain on some particular structure which is only stretched or pressed upon during one position of movement. The other patient in whom deviation in stooping continues to increase with the increase of the stooping, would probably be perfectly safe from the manipulative point of view, and would benefit from it.

The same sort of thing is seen in the shoulder. Here the patient raises the arm up to a certain point, the movement begins to become painful and is apparently going to be limited. Then by rotating the humerus the movement of elevation can be completed both in forward flexion and in abduction. On the return journey however the same rotation takes place in spite of the fact that there is not as yet definite impairment of movement. It is wise to regard this joint (or the sub-aromial bursa) as actively inflamed.

When it comes to deciding whether limitation of movement or pain referred from the joint is due to present active inflammation within the joint, or to pathological changes which have taken place as a result of a past inflammation which is now quiescent, all our diagnostic resources must be called to aid.

There is, however, one point in history which is of vast importance. When a sub-acute inflammation is still present, the patient will often give a story that, after rest, stiffness and pain are present. Then movement starts, it seems to loosen out the stiffened region and movement remains possible until fatigue sets in. These are the patients who say they are " no use" until they have loosened out in a hot bath after waking in the morning. On the other hand, if there has been past inflammation which is now quiescent, the story will be that rest eases, while movement never by any chance decreases the symptoms, which become steadily and progressively worse once they have started. Sometimes, of course, a past inflammation which is now quiescent will leave in its train a pathological change, and, if this causes deranged function, the ordinary use of the joint may keep alive a sub-acute inflammatory state.

X-ray examination. This is often of value in helping to decide whether inflammation is active or not, but the presence of joint changes in the photographs really means little or nothing from a manipulation point of view. If any shadow is shown which even so much as raises doubts as to the presence of tuberculosis or malignant disease, there is only one thing to be done and that is to withhold manipulative treatment of all sorts; the risk is far too great. The visible signs of inflammation having been present within the joint really means very little from our present point of view. It simply means that active inflammation has been present, but the decision as to whether it is still active or not depends on other methods of diagnosis. This much may be said with certainty, that we are in no way justified in regarding visible joint changes in an X-ray photograph as of necessity a contra-indication to manipulation. As has already been said, when these changes are noted, they simply indicate that inflammation has been present and of a sufficient severity or duration to leave its mark on cartilage and perhaps bone. No X-ray examination can tell us to what extent soft structures have been involved by the same inflammation that affected the cartilage and bone, and often enough the symptoms of which the patient complains are due to the effect of the inflammatory process on these structures, whereas the bony and cartilaginous changes are innocuous from the patient's point of view.

In these cases the breaking down of adhesions in the soft parts will often bring relief from symptoms even though the changes shown on the photographs 
may be gross. This particularly applies to the joints of the spine. Whereas we know gross changes are often present in men engaged in the most laborious occupations, yet they suffer no symptoms and no suspicion of joint change would be suspected, but for accidental disclosure during examination for some other condition.

The value of X-ray examination is to exclude gross disease or atrophy and to help in deciding a most important problem from the manipulation point of view-namely-whether the full range of movement is still attainable or not. There is no excuse whatever for manipulating a joint if there is any reasonable doubt as to whether active inflammation is present or not, or if the condition of the bone is not entirely satisfactory. A marked degree of osteoporosis often exists in most unexpected cases.

We now pass to a more difficult problem still and that is the question of whether the symptoms are due to an active inflammation which is now quiescent, but which has left behind a pathological change which is kept more or less actively inflamed as the result of function. There is only one way of finding out this and that is to place the joint at rest and see whether the signs of acute inflammation subside. If so, it is then very often justifiable to place the joint at rest until all symptoms have entirely disappeared for not less than about a fortnight. Then manipulation may lead through to complete success, whereas, had it been done without the preliminary rest, it might very easily have led through to equally complete failure. "Rest" means rest from function, not rest from painless movement which should be encouraged.

There is one other point worthy of consideration in trying to solve this knotty problem. Pain on movement as the result of active inflammation, and as the result of an old inflammation which is now quiescent, may be almost identical. In both there may be pain and limitation of movement in all directions. If the pain is confined to movement in one direction the chances are that active inflammation is not present; it is when all movements are painful and limited that the difficulty arises. It is always well to examine the corresponding joint of the opposite side as a preliminary. Then if movement is free and painless in the affected side up to a certain point, and if, when this point is reached, the symptom of pain is noticed and increases with increased range of movement, there is strong presumptive evidence that the trouble is due to the presence of a pathological condition which is now quiescent. If, however, pain is noticed at the beginning of movement, but with a little persuasion the range of painless movement increases, then as a rule we may take it for granted that active inflammation is still present.

Referred pain. Hitherto we have spoken freely about pain on movement without considering adequately what this expression implies. It must not be taken that pain, as a result of movement, is only referred to the particular joint itself. It is well known for instance that in cases of arthritis of the hip, pain is frequently noticed in the neighbourhood of the knee; while a similar condition of the shoulder joint or cases of inflammation of the sub-acromial bursa, show their symptoms in the form of pain radiating down the arm-a symptom most incorrectly attributed to brachial neuritis. The fact remains, however, that pain in the joint is very seldom confined to the joint itself. If it reaches any degree of severity the pain is usually felt to radiate over a very wide area. Hence it comes about that manipulation of the upper cervical spine for example may cause pain to radiate upwards over the back of the head and may often extend to the frontal aspect. In some patients in whom the symptoms are severe, movement of these joints will sometimes be noticed to have a direct effect upon the pupil. Pain 
referred from the region of the third or fourth cervical joints may send radiating pain along any of the branches of the cervical plexus, and particularly along the superficial branches. Posterior auricular, auriculo-temporal, and transverse facial neuritis (so called), are often merely symptoms of a pathological condition in these joints. If active inflammation is no longer present, cure of the symptom will follow manipulation.

We may be justified in referring to these symptoms as "rheumatic" in origin, but later we shall see that this need not necessarily be so. Should the condition of the joint cause irritation in the neighbourhood of the region of the phrenic nerve, spasm of the diaphragm may cause acute pain in the lower part of the chest and this may be mistaken for pleurisy.

Should the symptoms be derived from the region of the fifth and sixth the symptom may appear over the deltoid in the distribution of the circumflex which derives its origin from this region. The same story can be continued throughout the whole length of the spine.

\section{Manipulation.}

No attempt can be given here to enter into the details of technique. Suffice it to say that the manipulation of each individual joint requires a different technique, and the movements performed must of necessity be based entirely upon an accurate knowledge of the living anatomy of the joint and of any deviation from the normal which may be present. The point of this may be illustrated by considering manipulative treatment of the sacro-iliac joint. If a sacralised transverse process of the fifth lumbar vertebra is present, or if, without being sacralised, it is in very close proximity to the ala of the sacrum, the utmost care must be taken during the manipulation not to lordose the lumbar spine, indeed it should be kept flexed throughout the whole of the manipulation. The technique under these circumstances is far more difficult than if lordosis is allowed to take place. Still it is better to fail with the manipulation of the one joint than to inflict very severe injury upon the soft parts by nipping them between two bony points.

The next rule that should be imperative is that, in every joint that is subject to manipulation, the movements which are not under voluntary control should be restored before we make any attempt to restore those that are so.

The next vitally important point is that during manipulation the objective is to move one joint surface upon another. Therefore, in order to know exactly what we are doing, one joint surface must be fixed. The art of fixing is again one which only close anatomical study can teach, yet if it is not done, it is impossible to judge to what extent the mobilising force will be exerted at the one point where movement is desirable. Neglect of adequate fixation in this way is one of the common causes of failure of manipulation.

Another equally important point is that there is no excuse for an exhibition of great force. Indeed the shorter the leverage the safer the procedure. There are certain movements which are relatively safe, e.g. tension. There are other movements which involve leverage and here discretion is the better part of valour. Finally, when the mobilising force is applied, in no circumstances should the whole movement be carried through with anything resembling a swing. There is only one safe law: take up the slack, and then, and only then, push or pull.

The range of manipulation. Controversy has arisen, and to a certain extent does still arise as to how far we should attempt to secure the full range of movement at one sitting. This is a point where the only safe guide is experience. It 
is obvious however that, in the early stages of practice, the experience has to be won; and so some sort of guide on this very important point must be given. All it is possible to say is this. If there is any gross limitation of movement in any given direction, and particularly if this limitation has been present for any considerable time-shall we say for two months or more-it is usually wise to cease all movement after the first dead point has been passed. When this has been accomplished, if, as a result, the complete range of movement seems to be restored, well and good; but if on further movement, further impediment to movement is discovered, it should be left alone. Very much the same applies to a joint in which the movements are limited in more than one direction. Let us take the shoulder as an example. If gross limitation of movement has been present for any length of time, it is wise to warn the patient before starting that we shall not attempt to secure full range of movement at the first sitting. The chances are that during abduction (after movements which are not under voluntary control have been performed), some very definite obstruction to movement is overcome, abduction itself becomes more free and the safe thing to do then is to stop. The patient on recovering has little or no reaction, pain is negligible, advantage is at once taken of the increased range of movement, and, as function begets function, the range will be found to improve steadily, sometimes even passing through to complete recovery. Following this up by a second manipulation the abduction should be repeated and completed, and forward flexion should be restored. After any impediment to this movement has been overcome, and adequate time has been allowed to elapse, rotation should be freed by a third manipulation, if necessary. No definite time can be fixed as to when the second or third manipulation should be performed. Improvement in painless voluntary movement should follow any manipulation at once and should be progressive - when progress ceases, and not till then, are we at liberty to consider giving another dose of manipulation. More failure from manipulation is attributable to over zealousness than any other single cause. One particularly sad case comes to memory.

A patient had had an unusually severe attack of general rheumatoid arthritis following an acute infection some years previously. She spent her whole life sitting up, unable to feed herself because of lack of adequate flexion in the elbows. $\mathrm{X}$-ray examination of the joints showed the bone and cartilage surfaces comparatively slightly affected. I suggested manipulation of each joint in the manner described above. Being anxious for a change in environment before beginning this treatment, she went to visit friends in the neighbourhood of one of the Spas. Here she was told that this idea of slow restoration of movement was ridiculous, that it could all be done freely under one general anæsthetic and that recovery would be rapid. The patient died of shock in something just over forty-eight hours after the manipulation.

The idea underlying all manipulative treatment of a joint should be to secure an increase in the range of voluntary movement. Pain on movement inhibits movement, and therefore it is essential, if the manipulation is to be a success, that pain should not be a prominent feature: if it is, relapse is inevitable. This can only mean one of two things. Either the manipulation was performed on an unsuitable case, which means an error in diagnosis, or too much was attempted, which means an error in technique.

One more consideration is of vital importance, and that is the fact that, just as movement begets movement and function begets function, so too lack of movement destroys elasticity in all of us and particularly in those who have reached or 
and which for the most part are considered to be of rheumatic origin. Let us take one example - the effect of inadequacy of the ilio-tibial band. This band, in normal circumstances, allows a certain amount of movement of the crest of the ilium to take place. If for any reason the elasticity of the band is reduced, it must of necessity limit the movement of the ilium. This must in turn have an effect on the movements of the pelvis; and, as the pelvis moves upon the lumbo-sacral junction, limitation of the movement may cause irritation of the joints in question, and the final result will be interference with the normal function of those joints. Chronic backache of the low type will then be the main symptom, but the pain will be also felt down the leg, partly due directly to local tenderness of the band itself and partly referred from the joints as the result of interference with their proper function. Relief of the symptoms must depend on relief of the conditions causing them, and this must include restoring the full mobility of the joints. Thus it comes about that so many cases commonly attributed to rheumatism, but which are often not rheumatic in origin, can receive relief as a result of manipulation, and to refer to the condition as "rheumatic" is certainly to apply a misnomer.

To take another example, let us imagine that for some reason one of the joints of the thoracic spine, or one of the costo-vertebral joints, is not functioning properly. If, as a result of a break-down in muscle co-ordination, sudden strain is placed upon one of these joints, it can inflict upon these joints a condition similar to direct trauma. As a result, radiating pain may be felt throughout the whole of the distribution of the intercostal nerve concerned, and pain radiating from these sources may be felt throughout the distribution of the nerve in the walls - of thorax and abdomen. Symptoms may then arise which are confused with symptoms which might arise were the organs underneath these walls involved in some pathological condition. Here again, however, is another fertile source of error in diagnosis. The pain thus experienced is the counterpart of that which exists when a diagnosis such as traumatic lumbago is made. Lumbago is commonly supposed to infer that a rheumatic condition is present. Traumatic indicates that trauma is the origin of the symptoms. It is hardly conceivable that, in an otherwise prefectly healthy individual, some sudden strain should precipitate instantaneously an attack of rheumatism of the most acute possible type-so acute and so instantaneous that the patient may suffer from all the symptoms of severe shock. It is commonly said that cases of this type owe their origin to a torn muscle, but this is usually pure speculation, and the diagnosis is entirely unjustifiable unless, when the patient is in a condition to be examined, the symptoms can be reproduced by making the suspected muscle contract without movement of the joints in response to faradism. When this test is applied, it is surprising how very seldom any muscular lesion can be demonstrated. On the other hand the proof of the pudding is in the eating; and if, as a result of restoration of normal mobility by manipulation, symptoms are relieved-and this is a very common sequel-the diagnosis of muscular injury is proved to be without foundation.

Differential diagnosis of the type indicated is the outcome of the growth of physical medicine, and a considerable period of time must elapse before diagnosis of this type becomes general knowledge. It is however a lamentable fact that countless cases are treated daily for rheumatism and for prolonged periods of time, when no trace of rheumatism is actually present, but merely the result of joint strain or injury. How then is the difficulty to be overcome? There is surely only one way, and that is that, when a patient presents himself with symptoms that might possibly be attributable to rheumatism, but in whom the symptoms have arisen acutely and suddenly during some movement, an attempt should be made 
to examine the joints in the neighbourhood of the origin of the nerves that supply the part affected. This however is not all. It is right and proper that, when a patient is found to be suffering from a condition which might be diagnosed as neuritis, neuralgia and so forth, the ordinary rheumatic treatment should be given a chance. The thing that is not right and proper is that, if this treatment fails to give relief, further investigation should be withheld.

As has been said, differential diagnosis of an exact character may call for special examination of a highly technical description, but it is usually comparatively easy to establish that joint movement accentuates the symptoms. When this is established, the question of the advisability of manipulating the joints to restore their normal function becomes one of vital importance. The pros and cons have already been considered, so it comes about that any unexplained rheumatic condition which does not clear up, or at least tend to improve materially as a result of the ordinary rheumatic remedies, is worthy of further consideration ; and if movement of a joint is found to be limited, or to cause pain anywhere in the neighbourhood of the origin of the nerve trunk in which the pain is felt, the question of manipulation should always be considered. It is neglect of this practice that has led so many members of the general public to seek advice from medically unqualified practitioners of manipulation, and it is the success of manipulation at their hands that has established their reputation. The fact that medical men encounter the failures, and sometimes the disasters, of manipulation applied to unsuitable conditions, or of unskilled manipulation in suitable conditions, does not detract in the least from the number of those who receive benefit. The statement is probably true that the public hears of the successes of the unqualified practitioner but not of his failures-the successes are none the less there.

Once the possibility of the benefits to be secured from manipulation are realised, a very large number of cases at present attributed to rheumatism will be found to owe their origin to a lack of proper functioning within a joint, and often enough this is so, even though the patient himself may be quite unconscious of any actual disturbance of mobility in the joint until he is subjected to the test of putting it through the full range of physiological movement while the muscles are in a state of relaxation. Hence we see that, not only has manipulative treatment a place in the after-treatment of true rheumatic infection of the joints, but it also should play a prominent part in bringing relief to symptoms, sometimes labelled rheumatism or at least rheumatic in origin, whose true origin is some interference with normal joint function, which, in turn, is not attributable to rheumatism as this word should be understood. 